\title{
A Method of Measuring Gamma-Velocities of Contact Binary Stars
}

\author{
G. Kaszás ${ }^{1}$, J. Vinkó ${ }^{1}$ and G. Fürész \\ Department of Optics \& Quantum Electronics, JATE University, PO \\ Box 406, Szeged, H-6701 Hungary
}

\begin{abstract}
Gamma-velocities of bright contact binary stars (VW Cep, SW Lac, AB And, OO Aql) have been computed using cross-correlation. The template spectra were synthesized with a newly developed computer code WGMODEL.
\end{abstract}

\section{Introduction}

We have studied the radial velocities of some of the brightest northern contact binary stars using medium-resolution spectra. These radial velocities are useful to determine both the absolute dimensions of binary stars and their kinematic properties. The kinematics of these variable stars may contain important information on their galactic population and evolutionary state, which is still an unsolved mystery of binary star astrophysics.

\section{Observations}

The spectra were obtained with the Cassegrain spectrograph attached to the 1.88-m telescope of David Dunlap Observatory (Toronto, Canada). The spectra covered the $6500-6700 \AA$ wavelength interval including the $H \alpha$ region. The resolving power was $R=11,000$. The spectra were reduced with standard $I R A F$ routines including bias correction, flat fielding, cosmic ray removal and continuum normalization. Telluric lines were removed using observed telluric standard stars. The list of observed systems is shown in Table 1. In addition, we obtained spectra of several IAU standard velocity stars to determine the zero point of our velocity system (see Fig. 1).

\section{Method}

The $\gamma$-velocities of these binary systems were derived by cross-correlating the contact binary spectra with synthesized model line profiles computed by a newly developed computer code WGMODEL (Fig. 2). This method needs the parameters (inclination, mass ratio, fill-out factor, semi-major axis, period, tempera-

\footnotetext{
${ }^{1}$ Visiting observer, David Dunlap Observatory, Richmond Hill, Canada
} 
Table 1. The results of $\gamma$-velocity determination. The minimum, maximum, mean and scatter columns contain the statistical informations about radial velocities calculated using different standard stars.

\begin{tabular}{crrrrrrr} 
Star & $\begin{array}{r}\text { No. of } \\
\text { Spectra }\end{array}$ & $\begin{array}{r}\text { Period } \\
(\text { day })\end{array}$ & $\begin{array}{r}V_{\min } \\
(\mathrm{mag})\end{array}$ & $\begin{array}{r}\text { Minimum } \\
\gamma(\mathrm{km} / \mathrm{s})\end{array}$ & $\begin{array}{r}\text { Maximum } \\
\gamma(\mathrm{km} / \mathrm{s})\end{array}$ & $\begin{array}{r}\text { Mean } \gamma \\
(\mathrm{km} / \mathrm{s})\end{array}$ & $\begin{array}{r}\text { Scatter } \\
(\mathrm{km} / \mathrm{s})\end{array}$ \\
\hline VW Cep & 40 & 0.27831 & 7.7 & -16.76 & -14.64 & -15.69 & 0.54 \\
SW Lac & 20 & 0.32072 & 9.4 & -16.44 & -7.13 & -12.22 & 2.69 \\
AB And & 9 & 0.33189 & 10.3 & -24.99 & -17.12 & -20.30 & 2.85 \\
OO Aql & 9 & 0.50679 & 9.9 & -53.15 & -43.68 & -48.29 & 3.23
\end{tabular}

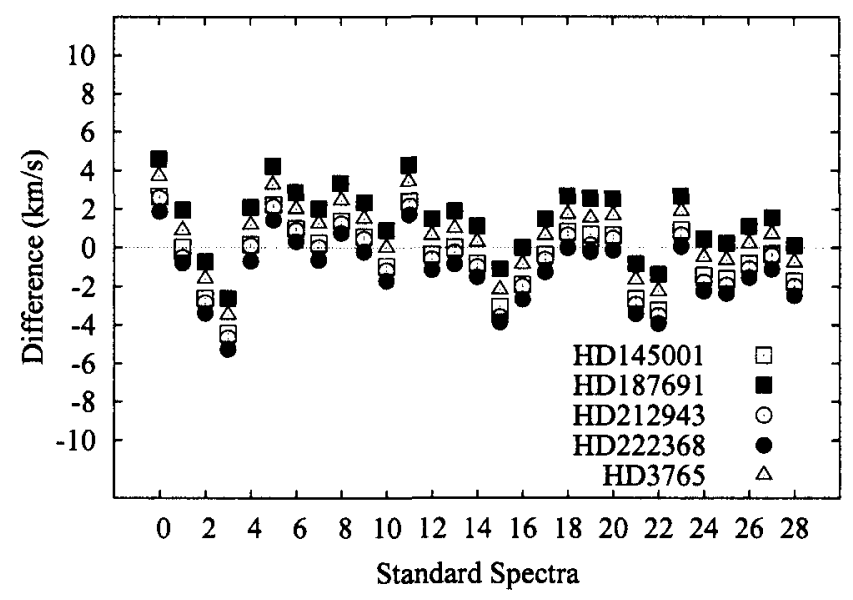

Figure 1. Velocities of selected bright stars with various spectral types have been determined with respect to several IAU standard velocity stars. These stars served as templates of the cross correlation in the synthesis method. This figure shows the deviation of the inferred velocities from the values given in the Bright Star Catalogue. 

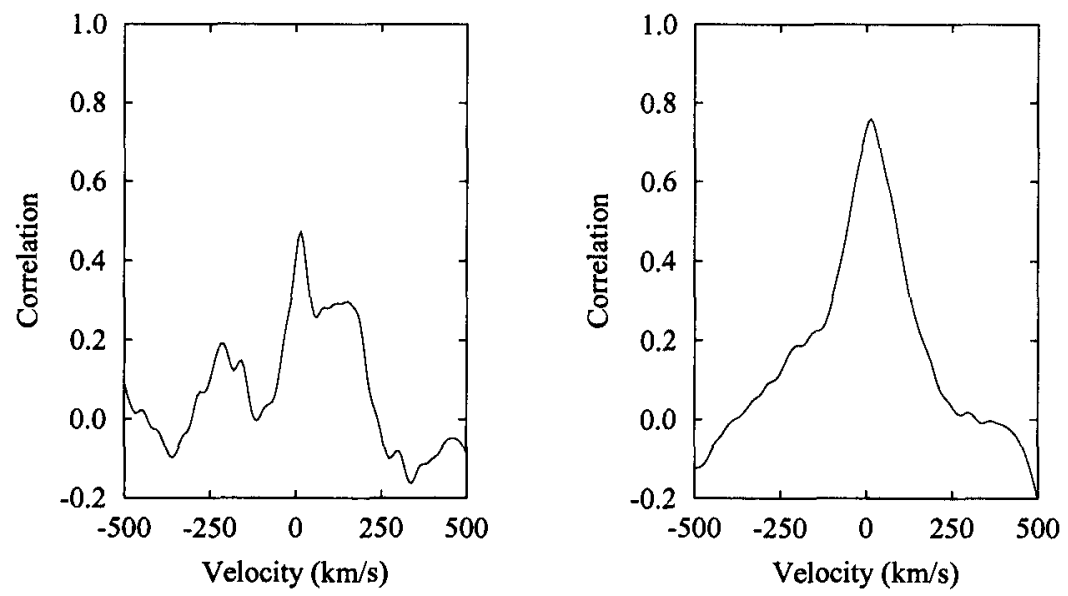

Figure 2. The cross correlation function obtained with "standard star" method (left panel) compared with the CCF calculated with synthesis method (right panel). The profile in the left panel is highly broadened and depends on orbital phase. Note the presence of the third light which further complicates the profile in the case of VW Cephei. Contrary to these, the CCF profile in the right panel is narrower, timeindependent and its maximum is at the value of $\gamma$-velocity. Averaging the velocities obtained from this CCF profiles at different orbital phases one can improve the accuracy of the final $\gamma$-velocity.

tures) of the contact binary. First, we synthesize the template spectrum using the calculated broadening profile of the contact binary and the standard star spectrum. Second, we calculate the cross-correlation of the contact binary and the synthesized template spectra. Third, we determine the $\gamma$-velocity from the maximum of the CCF-profile. This method gives the $\gamma$-velocity value independently from the actual orbital phase of the observed spectrum. We repeat these steps for each orbital phase of the contact binary and average the $\gamma$-velocities for the whole orbital cycle.

\section{Results}

Our tests showed that this method has internal accuracy of better than $2-3 \mathrm{~km} / \mathrm{s}$ (see Fig. 3), whereas the broadening of the individual line profiles caused by the orbital motion is larger than $200 \mathrm{~km} / \mathrm{s}$ in most cases. The averaged velocities of some of our program stars are collected in Table 1. The parameters of the systems are from Maceroni \& van't Veer (1996) and Kaszás et al. (1998). 


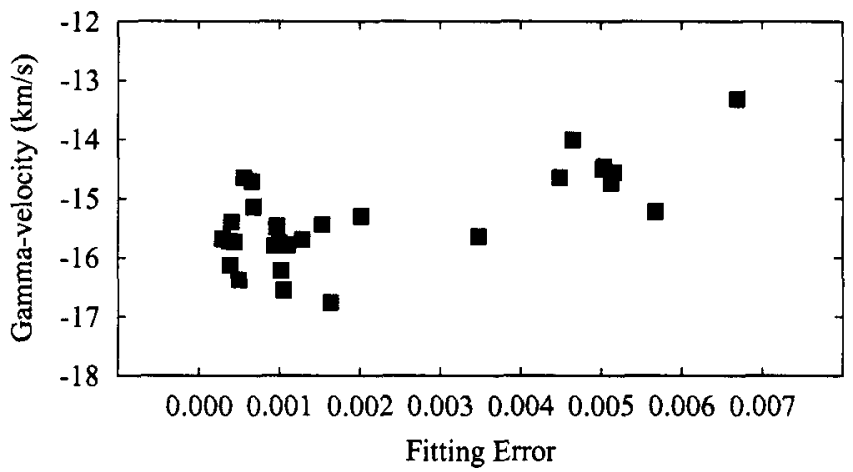

Figure 3. The spectral type of the selected standard star affects the precision of the inferred $\gamma$-velocity. The suitable velocities can be separated based on the goodness-of-fit of the observed and synthesized spectra. In the case plotted above (VW Cephei) each point represents a $\gamma$-velocity computed using a standard star with a given spectral type, averaged over the whole orbital cycle. The velocities having $\chi^{2}<0.003$ (dotted line) have been used in computing the final $\gamma$-velocity.

We conclude that the $\gamma$-velocities obtained with our method are adequate and useful for further studies to investigate the kinematic properties of $\mathrm{W}$ UMatype binary stars.

Acknowledgments. This work was funded partly by Hungarian OTKA Grants No. F022249, T022259, the Hungarian OMFB Grant No. Mec/P1988/98, and the Soros Foundation Travel Grant No. 222/2/3624. Thanks are also due to the staff of David Dunlap Observatory and Department of Astronomy, University of Toronto for kindly granting telescope time and assisting during the spectroscopic observations. Support from an IAU Travel Grant and the Organizing Commitee of this Colloquium is also gratefully acknowledged.

\section{References}

Maceroni, C., \& van't Veer, F. 1996, A\&A, 311, 523

Kaszás, G., Vinkó, J., Szatmáry, K., Hegedüs, T., Gál, J., Kiss, L.L., \& Borkovits, T. 1998, A\&A, 331, 231 